\title{
Anaphylaxis in Pregnancy
}

\author{
Thirukumar Markandu* (1), Kavurirel Eliyas Karunakaran \\ Department of Clinical Sciences, Faculty of Health-Care Sciences, Eastern University Sri Lanka, Batticaloa, Sri Lanka \\ Email: ^dr.thiru10@yahoo.com
}

How to cite this paper: Markandu, T. and Karunakaran, K.E. (2020) Anaphylaxis in Pregnancy. Open Journal of Obstetrics and Gynecology, 10, 698-707.

https://doi.org/10.4236/ojog.2020.1050063

Received: April 5, 2020

Accepted: May 10, 2020

Published: May 13, 2020

Copyright (c) 2020 by author(s) and Scientific Research Publishing Inc. This work is licensed under the Creative Commons Attribution International License (CC BY 4.0).

http://creativecommons.org/licenses/by/4.0/

\begin{abstract}
Anaphylaxis is a serious, generalized, hypersensitivity reaction which can be life threatening and one of the most serious medical emergencies. Anaphylaxis in pregnancy although rare, is attributed to the use of various drugs in pregnancy mainly following an administration of antibiotics. This review article describes several aspects of anaphylaxis such as aetiology, pathophysiology and management. Immediate recognition and initiation of treatment are the keys for satisfactory outcome. A multidisciplinary care and provision are other keys for achieving this good outcome.
\end{abstract}

\section{Keywords}

Anaphylaxis, Pregnancy, Multidisciplinary Care

\section{Introduction}

Anaphylaxis is a serious, generalized, hypersensitivity reaction which can be life-threatening [1]. It is one of the most serious medical emergencies, where rapid diagnosis and prompt and appropriate treatment save lives.

A prospective population-based study on anaphylaxis in pregnancy by $\mathrm{Mc}$ Call et al. [2], has shown that anaphylaxis is a rare but potentially serious event in terms of maternal and fetal outcomes. It is frequently the result of a reaction to antibiotic administration mostly because of the prophylactic use of antibiotics at the time of a cesarean section [CS] [2].

The pregnant and the non-pregnant share similar aetiology, patho-physiology and management principles. This review article describes anaphylaxis in general which is applicable to both pregnant and non-pregnant population.

A search strategy using appropriate medical subject headings was developed. Electronic databases (PubMed, Popline, Cochrane, CINAHL, Embase, and ClinicalTrials.gov) were searched from inception through January 2019. Case studies, descriptive studies and guidelines from various professional bodies were in- 
cluded.

\section{Aetiology}

Anaphylaxis in pregnancy is considered a rare condition with an estimated prevalence of 2.7 cases $/ 100,000$ deliveries. Both pregnancy and non-pregnancy share the same aetiology for anaphylaxis [1]. The anaphylaxis during pregnancy occurs most commonly due to antibiotics and nonsteroidal anti-inflammatory medications; other medications, such as intravenous iron and intravenous vitamins B1, B6, and B12 which are used for the treatment of hyperemesis gravidarum in some countries. Among antibiotics most commonly due to penicillin and other beta-lactam antibiotics administrated in intrapartum for prevention of neonatal group B Streptococcal (GBS) infection or prevention of maternal infection after CS [3] [4]. Other than antibiotics, oxytocin, neuromuscular blockers, epidural medications, general anaesthetics, natural rubber latex and chlorhexidine have also been implicated [5]. Medications used in pregnancy such as ranitidine, snake antivenom are less commonly associated with anaphylaxis, so does bee sting [3].

Exercise during pregnancy rarely induces anaphylaxis. During puerperium, maternal anaphylaxis occurs in the first 3 days of postpartum probably due to rapidly declining serum progesterone level following placental delivery. The use of non-steroidal anti-inflammatory agents can also precipitate anaphylaxis during breast-feeding [6].

\section{Pathophysiology}

There are two pathways for anaphylaxis

1) IgE-dependent anaphylaxis

2) IgE-independent anaphylaxis

1) IgE-dependent anaphylaxis

It typically occurs through an IgE-mediated mechanism leading to mast cell activation. The IgE antibodies do not cross the placenta and therefore, fetus is relatively protected from the ill effect of anaphylaxis [7].

IgE is found at the lowest concentrations in the circulation. However, the level is higher in individuals with atopic diseases [8] [9]. IgE binds to the high affinity receptor FceRI on the surface of basophils, mast cells, and other cell types, such as neutrophils, eosinophils, monocytes dendritic cells and platelets [10]. Upon exposure to a bi or multi-valent allergen, crosslinking of FceRI-bound IgE induces activation of mast cells and basophils and the immediate release of preformed mediators such as histamine and various proteases promotes the synthesis of leukotrienes, prostaglandins and cytokines [9].

\section{2) IgE-independent anaphylaxis}

Anaphylaxis was also observed in a case following infusion of large quantities of a drug or a monoclonal antibody. Therefore, it is believed that there can be an IgE-independent pathway of anaphylaxis. It is demonstrated in mice that 
IgG-mediated anaphylaxis needs a larger dose of antigen than that of IgE-mediated anaphylaxis [11]. It was also noted that anaphylaxis can occur in individuals who have undetectable levels of IgE in their circulation [12] indicating IgE independent anaphylaxis.

\section{Role of complement}

Many stimuli activate compliment system during anaphylaxis results in formation of anaphylatoxins such as C3a, C4a, and C5a, which are potent inflammatory mediators [13]. The evidence suggests that anaphylatoxins might be involved in anaphylaxis.

\section{The Patterns of Anaphylaxis Reaction}

Anaphylaxis can be one of the three patterns, such as uniphasic, protracted and biphasic [14]. The uniphasic anaphylaxis response usually peaks within 30 to 60 minutes following the exposure and the symptoms resolves with or without treatment within an hour. This is the most common type of anaphylaxis and it occurs in 80 to 90 percent of cases. The protracted anaphylactic reaction lasts hours to days without complete resolution. They are uncommon and only few cases were reported [15].

The Biphasic anaphylaxis is characterized by a uniphasic response, followed by an asymptomatic period of an hour or more and subsequently the symptoms reappear without further exposure to antigen [16]. It occurs in 10 to 23 percent of all anaphylactic reactions [17]. The severity of recurrent symptoms is unpredictable and the second phase of symptoms does not necessarily resemble the initial symptoms [18]. However, the recurrent symptoms are usually less severe than the initial symptoms [17].

Sometimes, the second phase of an anaphylaxis episode can be more severe or even fatal [19]. Urticaria seems to be particularly common and often the only symptom during the second phase. The period between resolution of initial symptoms and onset of recurrent symptoms varies from 1 to 72 hours [20].

Several theories have been proposed to explain the biphasic nature of the anaphylaxis. One school of thought is that it is due to the influx of inflammatory cells in response to cytokines and chemotactic factors released during the initial response which is due to mast cell degranulation that is responsible for the initial symptoms [19].

However, the histological findings in biphasic anaphylaxis do not support the above theory. Other school of thought is that the biphasic response is related to a second wave of mast cell degranulation. Another theory implicates uneven antigen absorption [19].

It is impossible to predict which patients will develop a biphasic response. Severe initial symptoms might be a risk factor. Specifically, the need for more than one dose of adrenaline or intravenous fluids for hypotension was noted [20]. Delayed administration of adrenaline when initial symptoms appear or suboptimal treatment of initial reaction might increase the risk of biphasic reaction 
[20].

Further, studies have found no evidence that glucocorticoids reduce the rates of biphasic reactions [21]. The use of beta-adrenergic blockers, older age and concomitant cardiovascular disease can also predispose biphasic responses [22]. Since the biphasic nature is unpredictable even though patients with anaphylaxis that resolved promptly and completely with treatment, Individuals with severe anaphylaxis should be admitted to an observation unit for at least 24 hours.

\section{Diagnosis and Differential Diagnosis}

Anaphylaxis manifests as an acute onset illness with typical skin features such as urticarial rash, erythema, angioedema with systemic symptoms such as respiratory symptoms (wheeze, dyspnea), cardiovascular (feeling faint) and gastrointestinal symptoms (abdominal pain, vomiting). However, symptom patterns can vary. An acute cardio respiratory compromise can occur in the absence of skin features or only a few symptoms might be present during a given episode [23].

In the case of anaphylaxis, skin and mucosal involvement is observed in $80 \%$ to $90 \%$, respiratory tract involvement in up to $70 \%$, gastrointestinal tract involvement in up to $45 \%$, cardiovascular involvement in up to $45 \%$, and central nervous system involvement in up to $15 \%$. Sudden onset of symptoms and signs within minutes to few hours after exposure to allergen is characteristic of anaphylaxis. When it progresses rapidly, the clinical diagnosis of anaphylaxis can be arrived, and management must be initiated without delay for optimal outcome [24].

The clinical presentations of anaphylaxis in pregnancy can be vulvar and vaginal itching, low backpain, uterine cramps, reduced fetal movement, and preterm labor (Table 1) [25]. The hypoxemia and hypotension have serious maternal effects on pregnancy in anaphylaxis. The fetus is also at risk for intrapartum asphyxia in maternal hypoxemia. The fetus can be subjected to hypoxic-ischemic encephalopathy, severe central nervous system damage or even death [26].

Within few minutes after the initial symptoms of anaphylaxis, mature tryptase released from mast cells and basophils can be detected in serum [27]. Thus, clinical diagnosis can be confirmed based on increased serum total tryptase levels preferably blood samples taken within 15 minutes to 3 hours after the onset of symptoms. Serial measurements (e.g., at presentation, 1 - 2 hours later, and at resolution) increase the sensitivity of the test [28]. It is worth to note that this laboratory assay is not helpful with initial diagnosis and management, although the results can be useful later.

The differential diagnosis of anaphylaxis, during pregnancy and in non-pregnancy status are acute asthma, acute generalized urticaria, acute angioedema, syncope (fainting), and acute panic or anxiety attack [29]; however, many other diagnoses, including mastocytosis need to be considered [30].

Clinical conditions causing maternal cardio-respiratory compromise should be considered into the differential diagnosis during intrapartum. Those conditions 
Table 1. Clinical criteria for diagnosing anaphylaxis. Anaphylaxis is highly likely when any one of the following three criteria is fulfilled.

1) Acute onset of an illness (minutes to several hours) with involvement of the skin, mucosal tissue or both (e.g. generalized hives, pruritus or flushing and swollen lips-tongue-uvula) and at least one of the following:
a) Respiratory compromise (e.g. dyspnea, wheeze-bronchospasm, stridor, reduced PEF and hypoxemia)
b) Reduced blood pressure (BP) or associated symptoms of end-organ dysfunction (e.g. hypotonia/collapse, syncope and incontinence)

2) Two or more of the following that occur rapidly after exposure to a likely allergen for that patient (minutes to several hours):
a) Involvement of the skin-mucosal tissue (e.g. generalized hives, itch-flush and swollen lips-tongue-uvula)
b) Respiratory compromise (e.g. dyspnea, wheeze bronchospasm, stridor, reduced PEF and hypoxemia)
c) Reduced BP or associated symptoms (e.g. hypotonia/collapse, syncope and incontinence)
d) Persistent gastrointestinal symptoms (e.g. cramping abdominal pain, vomiting)

3) Reduced BP after exposure to a known allergen for that patient (minutes to several hours):

a) Infants and children: low systolic BP (age-specific) or greater than $30 \%$ decrease in systolic BP.

b) Adults: systolic BP of less than $90 \mathrm{~mm} \mathrm{Hg}$ or greater than $30 \%$ decrease from that person's baseline.

$\mathrm{BP}=$ blood pressure: $\mathrm{PEF}=$ peak expiratory flow.

are pulmonary embolism, pulmonary edema, cardiomyopathy, acute coronary syndrome, mitral stenosis, hypotension, cerebrovascular accident, and amniotic fluid embolism (AFE) [31].

Maternal anaphylaxis constitutes a major concern for obstetricians and neonatologists. An alteration in immunological status due to increased progesterone level during pregnancy may predispose pregnant women to anaphylaxis even though the high levels of placental histaminase may act as a protective mechanism for the fetus [32].

\section{Severity of Anaphylaxis}

Following grading scale for severity of anaphylaxis is used.

Mild (Grade 1) anaphylaxis presents with mucocutaneous signs only, such as erythema, urticaria, and peripheral angioedema. The mild anaphylaxis (Grade 1) must be recognized and monitored carefully to detect the disease progression to higher grade reaction which is best managed with adrenaline.

Moderate (Grade 2) anaphylaxis has typically multi-organ manifestations such as mucocutaneous signs combined with hypotension and/or bronchospasm.

It may also have additional gastrointestinal or central nervous system symptoms and signs such as rhinorrhoea, cough, dyspnoea, circumoral tingling, difficulty in swallowing, nausea, abdominal pain, irritability, confusion or a sense 
of impending doom.

Life threatening (Grade 3) anaphylaxis is a clinical presentation of life-threatening hypotension and/or high airway pressure. Immediate treatment is required in this situation to avoid progression from inadequate tissue perfusion to cardiac arrest or significant hypoxia. The airway pressures are elevated to levels where oxygenation and ventilation are rapidly compromised. In up to 20 percent of patients only one of the signs of anaphylaxis is present [12].

Unlike Grade 2 anaphylaxis, cutaneous signs are frequently absent initially due to the low cardiac output and may appear only when circulation is restored.

Cardiac arrest (Grade 4) anaphylaxis is characterized by either absence of a palpable central pulse or a grossly inadequate blood pressure as assessed via direct arterial measurement.

\section{Management}

The management of anaphylaxis in pregnant patients is basically the same as that in non-pregnant ones. The management of patient with anaphylaxis should begin immediately upon the mere suspicion of this condition. Multi-disciplinary approach with intensive care support improves the likelihood of survival.

\section{Basic initial treatment}

One of the initial steps would be to call for help. The trigger, for instance intravenous drugs should be discontinued. Her airway, breathing, circulation, mental status and skin are assessed. Adrenaline should be promptly injected intramuscularly in the deltoid muscle or mid-outer thigh a dose of $0.5 \mathrm{mg}$ (half vial of $1 \mathrm{ml})(1: 1000)$ solution. Same dose can be repeated every 5 to 15 minutes, depending on the clinical response.

Adrenaline is the first medication to be administered when a patient develops anaphylaxis and it should not be delayed by taking time to administer second-line drugs with no immediate life-saving benefit, such as H1-antihistamines, H2-antihistamines, or glucocorticoids [33].

Adrenaline acts on alpha 1, beta 1 and beta 2 adrenergic receptors. Its action on alpha 1 adrenergic receptor is to induce vasoconstriction, which decreases tissue/airway edema, hypotension, and distributive shock; beta 1 adrenergic receptors to increase heart rate and cardiac contractility; and b2-adrenergic receptors to dilate the airways [34]. In addition, adrenaline blocks mast cells from releasing the mediators such histamine and eicosanoids [35].

The patient shall be kept on left lateral position to prevent added positional hypotension and lower extremities elevated. Manual displacement of the gravid uterus to the left might be necessary. Positioning on the back might lead to inferior vena caval compression and reduced venous return to the heart. If the patient is dyspneic or vomiting, comfortable position is required. It is important to avoid sitting or standing position as it can lead to cardiac arrest caused by the empty inferior vena cava/empty ventricle syndrome. Thus, fatality can occur within seconds if she stands or sits suddenly.

\section{Comprehensive care}


Maintaining adequate intravascular volume and oxygenation in the management of anaphylaxis during pregnancy are essential to prevent both maternal and fetal complications. Therefore, establish intravenous access with two 14- to 16-gauge cannula and commencement of rapid infusion of $0.9 \%$ isotonic saline within the first 5 - 10 minutes, are required. Administer 5 to $10 \mathrm{ml} / \mathrm{kg} 0.9 \%$ saline rapidly; large intravenous fluid volumes might be needed (e.g. $7 \mathrm{~L}$ ).

Administration of 100\% high-flow humidified supplemental oxygen 6 - 8 liters/minute with face mask or oropharyngeal airway is also required.

Continuous noninvasive electronic monitoring of maternal blood pressure, heart rate, oxygenation (by using pulse oximetry), and fetal heart rate with continuous electronic fetal monitoring should be instituted. If continuous electronic monitoring is not available, monitor maternal vital signs and fetal heart rate every 5 minutes or more frequently. Maintain a minimum maternal systolic blood pressure of $90 \mathrm{~mm} \mathrm{Hg}$ to ensure adequate placental perfusion.

When indicated at any time, prepare to perform cardiopulmonary resuscitations, initiating continuous chest compressions before rescue breathing. Compressions should be started at a rate of 100 to 120 per minute and a depth of 5 to $6 \mathrm{~cm}$ before giving rescue breaths. High-quality chest compressions can be difficult to perform in a fully gravid woman.

When indicated at any time, perform emergency cesarean delivery for anaphylaxis refractory to medical management as outlined above or for fetal distress [32].

Other therapies should be considered as second-line therapies and not a substitute for adrenaline (epinephrine). H1- and H2-antihistamines might be helpful in treating cutaneous and upper respiratory signs and symptoms, and corticosteroids might help prevent biphasic reactions but neither prevent nor treat airway obstruction or circulatory collapse and therefore cannot be considered alternatives to adrenaline [35].

\section{Anaphylaxis refractory to basic initial treatment}

Continuous intravenous infusion adrenaline may be required in patients with hypotension or shock refractory to basic initial treatment. The frequent dose of adrenaline needs titration based on continuous noninvasive monitoring of heart rate, blood pressure and oxygenation. This is best done by a team of anesthetists in intensive care unit set up.

\section{Conclusion}

Anaphylaxis is a rare occurrence but fatal. It requires immediate recognition and initiation of treatment and it should begin immediately upon the mere suspicion of this condition for satisfactory outcome. Adrenaline is the first medication to be administered without delay when a patient develops anaphylaxis. A multidisciplinary care is another key for achieving a good outcome.

\section{Conflicts of Interest}

The authors declare no conflicts of interest regarding the publication of this paper. 


\section{References}

[1] Simons, F.E.R. and Schatz, M. (2012) Anaphylaxis during Pregnancy. Journal of Allergy and Clinical Immunology, 130, 597-606. https://doi.org/10.1016/j.jaci.2012.06.035

[2] McCall, S.J., Bunch, K.J., Brocklehurst, P., D’Arcy, R., Hinshaw, K., Kurinczuk, J.J., Lucas, D.N., Stenson, B., Tuffnell, D.J. and Knight, M. (2018) The Incidence, Characteristics, Management and Outcomes of Anaphylaxis in Pregnancy: A Population-Based Descriptive Study. BJOG, 125, 965-971.

https://doi.org/10.1111/1471-0528.15041

[3] Chaudhuri, K., Gonzales, J., Jesurun, C.A., Ambat, M.T. and Mandal-Chaudhuri, S. (2008) Anaphylactic Shock in Pregnancy: A Case Study and Review of the Literature. International Journal of Obstetric Anesthesia, 17, 350-357. https://doi.org/10.1016/j.ijoa.2008.05.002

[4] Mulla, Z.D., Ebrahim, M.S. and Gonzalez, J.L. (2010) Anaphylaxis in the Obstetric Patient: Analysis of a Statewide Hospital Discharge Database. Annals of Allergy, Asthma \& Immunology, 104, 55-59. https://doi.org/10.1016/j.anai.2009.11.005

[5] Draisci, G., Zanfini, B.A., Nucera, E., Catarci, S., Sangregorio, R., Schiavino, D., et al. (2011) Latex Sensitization: A Special Risk for the Obstetric Population? Anesthesiology, 114, 565-569. https://doi.org/10.1097/ALN.0b013e318206ff50

[6] Baraka, A. and Sfeir, S. (1980) Anaphylactic Cardiac Arrest in a Parturient. Response of the Newborn. JAMA, 243, 1745.

https://doi.org/10.1001/jama.1980.03300430047025

[7] Dullaers, M., De Bruyne, R., Ramadani, F., Gould, H.J., Gevaert, P. and Lambrecht, B.N. (2012) The Who, Where, and When of IgE in Allergic Airway Disease. Journal of Allergy and Clinical Immunology, 129, 635-645.

https://doi.org/10.1016/j.jaci.2011.10.029

[8] Galli, S.J. and Tsai, M. (2012) IgE and Mast Cells in Allergic Disease. Nature Medicine, 18, 693-704. https://doi.org/10.1038/nm.2755

[9] Oettgen, H.C. (2016) Fifty Years Later: Emerging Functions of IgE Antibodies in Host Defense, Immune Regulation, and Allergic Diseases. Journal of Allergy and Clinical Immunology, 137, 1631-1645. https://doi.org/10.1016/j.jaci.2016.04.009

[10] Simons, F.E., Frew, A.J., Ansotegui, I.J., Bochner, B.S., Golden, D.B., Finkelman, F.D., et al. (2007) Risk Assessment in Anaphylaxis: Current and Future Approaches. Journal of Allergy and Clinical Immunology, 120, S2-24. https://doi.org/10.1016/j.jaci.2007.05.001

[11] Klos, A., Tenner, A.J., Johswich, K.O., Ager, R.R., Reis, E.S. and Kohl, J. (2009) The Role of the Anaphylatoxins in Health and Disease. Molecular Immunology, 46, 2753-2766. https://doi.org/10.1016/j.molimm.2009.04.027

[12] Zisa, G., Riccobono, F., Calamari, A.M., et al. (2009) A Case of Protracted Hypotension as Unique Symptom of a Biphasic Anaphylaxis to Amoxicillin. European Annals of Allergy and Clinical Immunology, 41, 60.

[13] Vinuya, R.Z., Simon, M.R. and Schwartz, L.B. (1994) Elevated Serum Tryptase Levels in a Patient with Protracted Anaphylaxis. Annals of Allergy, 73, 232.

[14] Scranton, S.E., Gonzalez, E.G. and Waibel, K.H. (2009) Incidence and Characteristics of Biphasic Reactions after Allergen Immunotherapy. Journal of Allergy and Clinical Immunology, 123, 493-498. https://doi.org/10.1016/j.jaci.2008.10.026

[15] Popa, V.T. and Lerner, S.A. (1984) Biphasic Systemic Anaphylactic Reaction: Three Illustrative Cases. Annals of Allergy, 53, 151-155. 
[16] Forrest-Hay, A., Taylor, C. and Tolchard, S. (2003) Biphasic Anaphylaxis in a UK Emergency Department. Open Paper Presentations of the 2003 Scientific Symposium of the Resuscitation Council of the United Kingdom (Abstract).

[17] Gould, H.J. and Sutton, B.J. (2008) IgE in Allergy and Asthma Today. Nature Reviews Immunology, 8, 205-217. https://doi.org/10.1038/nri2273

[18] Platts-Mills, T.A., Schuyler, A.J., Erwin, E.A., Commins, S.P. and Woodfolk, J.A. (2016) IgE in the Diagnosis and Treatment of Allergic Disease. Journal of Allergy and Clinical Immunology, 137, 1662-1670. https://doi.org/10.1016/j.jaci.2016.04.010

[19] Kraft, S. and Kinet, J.P. (2007) New Developments in Fcepsilon RI Regulation, Function and Inhibition. Nature Reviews Immunology, 7, 365-378. https://doi.org/10.1038/nri2072

[20] Strait, R.T., Morris, S.C. and Finkelman, F.D. (2006) IgG-Blocking Antibodies Inhibit IgE Mediated Anaphylaxis in Vivo through Both Antigen Interception and Fc Gamma RIIb Cross-Linking. Journal of Clinical Investigation, 116, 833-841. https://doi.org/10.1172/JCI25575

[21] Heinly, T.L. and Lieberman, P. (2000) Anaphylaxis in Pregnancy. Immunology and Allergy Clinics of North America, 20, 83-88. https://doi.org/10.1016/S0889-8561(05)70185-5

[22] Mertes, P.M., Malinovsky, J.M., Jouffrou, L., et al. (2011) Reducing the Risk of Anaphylaxis during Anesthesia: 2011 Updated for Clinical Practice. Immunology and Allergy Clinics of North America, 21, 442-453.

http://www.jiaci.org/issues/vol21 issue6/2.pdf

[23] Sampson, H.A., Munoz-Furlong, A., Campbell, R.L., Adkinson, N.F., Bock, S.A., Branum, A., et al. (2006) Second Symposium on the Definition and Management of Anaphylaxis: Summary Report-Second National Institute of Allergy and Infectious Disease/Food Allergy and Anaphylaxis Network Symposium. Journal of Allergy and Clinical Immunology, 117, 391-397. https://doi.org/10.1016/j.jaci.2005.12.1303

[24] Association Anaesthetists of Great Britain and Ireland (2009) Suspected Anaphylactic Reaction Associated with Anaesthesia. Anaesthesia, 64, 199-211.

[25] Braveman, F.R. (2008) Pregnancy-Associated Diseases. In: Hines, R.L. and Marschall, K.E., Eds., Stoelting's Anesthesia and Coexisting Diseases, 5th Edition, Churchill Livingstone, Philadelphia, 557-580. https://doi.org/10.1016/B978-1-4160-3998-3.10023-5

[26] Berardi, A., Rossi, K., Cavalleri, F., et al. (2004) Maternal Anaphylaxis and Fetal Brain Damage after Intrapartum Chemoprophylaxis. Journal of Perinatal Medicine, 32, 375-377. https://doi.org/10.1515/JPM.2004.070

[27] Berenguer, A., Couto, A., Brites, V. and Fernandes, R. (2013) Anaphylaxis in Pregnancy: A Rare Cause of Neonatal Mortality. BMJ Case Reports, 2013, bcr2012007055. http://www.ncbi.nlm.nih.gov/pmc/articles/PMC3603634

[28] Kroigaard, M., et al. (2007) Scandinavian Clinical Practice Guidelines on Diagnosis, Management and Follow up of Anaphylaxis during Anaesthesia. Acta Anaesthesiologica Scandinavica, 51, 655-670. https://doi.org/10.1111/j.1399-6576.2007.01313.x

[29] Schatz, M. and Dombrowski, M.P. (2009) Clinical Practice. Asthma in Pregnancy. The New England Journal of Medicine, 360, 1862-1869. https://doi.org/10.1056/NEJMcp0809942

[30] Caballero, T., Farkas, H., Bouillet, L., Bowen, T., Gompel, A., Fagerberg, C., et al. (2012) International Consensus and Practical Guidelines on the Gynecologic and Obstetric Management of Female Patients with Hereditary Angioedema Caused by 
C1 Inhibitor Deficiency. Journal of Allergy and Clinical Immunology, 129, 308-320. https://doi.org/10.1016/j.jaci.2011.11.025

[31] Czaller, I., Visy, B., Csuka, D., Fust, G., Toth, F. and Farkas, H. (2010) The Natural History of Hereditary Angioedema and the Impact of Treatment with Human C1-Inhibitor Concentrate during Pregnancy: A Long-Term Survey. European Journal of Obstetrics \& Gynecology and Reproductive Biology, 152, 44-49. https://doi.org/10.1016/j.ejogrb.2010.05.008

[32] Kishimoto, T.K., Viswanathan, K., Ganguly, T., Elankumaran, S., Smith, S., Pelzer, K., et al. (2008) Contaminated Heparin Associated with Adverse Clinical Events and Activation of the Contact System. The New England Journal of Medicine, 358, 2457-2467. https://doi.org/10.1056/NEJMoa0803200

[33] Clarke, R.C., Sadleir, P.H.M. and Platt, P.R. (2012) The Role of Sugammadex in the Development and Modification of an Allergic Response to Rocuronium: Evidence from a Cutaneous Model. Anaesthesia, 67, 266-273. https://doi.org/10.1111/j.1365-2044.2011.06995.x

[34] Australasian Society of Clinical Immunology and Allergy (2011) Anaphylaxis: Emergency Management for Health Professionals (Poster). Australian Prescriber, 34, 124. http://www.australianprescriber.com/magazine/34/4/artid/1210

[35] Muraro, A., Roberts, G., Worm, M., Bilo, M.B., Brockow, K., Fernandez Rivas, M., et al. (2014) Anaphylaxis: Guidelines from the European Academy of Allergy and Clinical Immunology. Allergy, 69, 1026-1045. https://doi.org/10.1111/all.12437 